\title{
QUANTITATIVE ASSESSMENT OF SURFACE ROUGHNESS USING BACKSCATTERED ULTRASOUND: THE EFFECTS OF FINITE SURFACE CURVATURE
}

\author{
Edward H. Chiang, ${ }^{\dagger}$ Ronald S. Adler, ${ }^{\dagger}$ Charles R. Meyer, ${ }^{\dagger}$ \\ Jonathan M. Rubin, ${ }^{\dagger}$ DAle K. DEDRICK ${ }^{\ddagger}$ and Timothy J. LAING* \\ ${ }^{\dagger}$ Department of Radiology, ${ }^{\ddagger}$ Orthopaedic Research Laboratories, Departments of Surgery (Orthopaedics Section) \\ and Internal Medicine (Rheumatology Division) and *Department of Internal Medicine (Rheumatology \\ Division), University of Michigan Medical Center, 1500 E. Medical Center Drive, \\ Ann Arbor, MI 48109-0030, USA
}

(Received 19 April 1993; in final form 20 September 1993)

\begin{abstract}
We have previously described a technique to quantify surface fibrillatory changes in osteoarthritic articular cartilage. In that study, the angular distribution of the scattered acoustic field from an insonifying source directly related to the distribution of surface fibrillatory changes. In the current study, we demonstrate a more sensitive method to quantify surface roughness, the effect of global surface curvature in estimating surface roughness and the utility of using focused transducers in circumventing this potential problem for in vivo work. Phantoms composed of acrylic rods with and without sandpaper grit (about 15 to $72 \mu \mathrm{m}$, mean particle size) applied to the surface were scanned. A more robust angular scattering technique to measure the angle dependent data was employed, in which the integrated squared pressure amplitude over a finite time window (mean power) was measured as a function of incident acoustic angle for varying surface roughnesses and radii of curvature. We show that the potential dynamic range for making roughness discriminations diminishes with decreasing radius of curvature of the acrylic rod phantoms using an unfocused transducer. This effect is minimized with use of a focused transducer. Roughness effects are most evident at sufficiently large angles where incoherent scattering dominates. We conclude that the roughness of cylindrically curved surfaces can be quantitatively assessed using a focused ultrasound beam at sufficiently large incident angles, given that the focal spot size is sufficiently smaller than the radius of curvature of the surface.
\end{abstract}

Key Words: Acoustics, Ultrasound, Ultrasonic scanning, Ultrasonic backscatter, Roughness, Surface curvature, Articular cartilage roughness, Osteoarthritis.

\section{INTRODUCTION}

The study of acoustical scattering of rough surfaces dates back to the time of Lord Rayleigh (Rayleigh 1945). Since then, extensive work has been done regarding the scattering of acoustic radiation from both periodically and randomly rough surfaces (Thorne and Pace 1984; Quentin et al. 1975; De Billy et al. 1976; Lynch 1970; Bozma and Kuc 1991). It has been shown that the angular distribution of scattered, acoustic radiation from a planar surface is directly related to variations in surface height, in a predictable manner. If the surface fibrillations are sufficiently small relative to

Address correspondence to: Ronald S. Adler, M.D., Ph.D. the acoustic wavelength, then the angular scattering is a direct measure of surface root-mean-square roughness (Thorne and Pace 1984), in which case, the Rayleigh-Rice approximation applies (Thorsos 1988). Another theoretical approach to acoustic scattering from rough surfaces is the Kirchhoff approximation, which is used when the radius of curvature is large compared to wavelength (Thorsos 1988).

Our initial ultrasonic study of osteoarthritic articular cartilage demonstrated that the angular distribution of the envelope detected backscattered pressure field from a focused ultrasonic transducer is directly related to the distribution of surface fibrillatory changes (Adler et al. 1992). Such fibrillatory changes are considered to be one of the earliest manifestations of osteoarthritis. No known technique currently exists to study these 


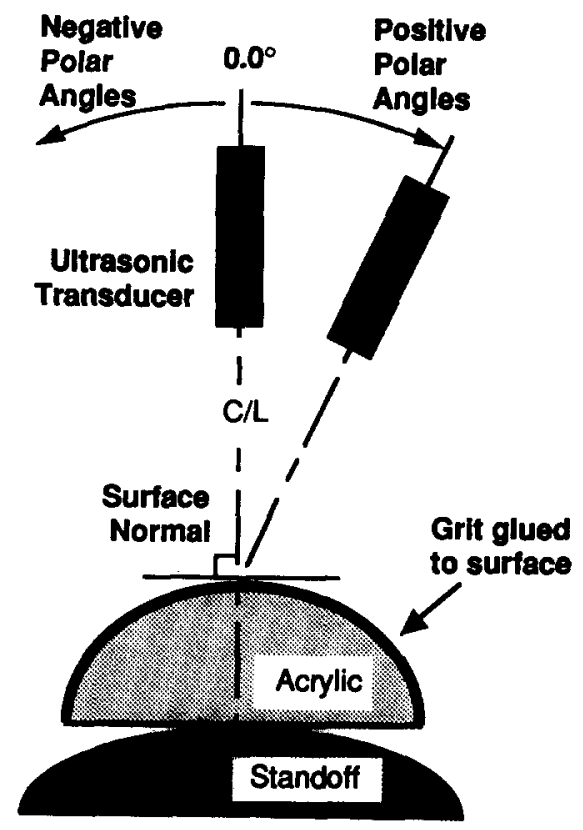

Fig. 1. Diagram showing polar angle. Polar angle, $\theta$, is define relative to the normal incident angle. Clockwise rotations are arbitrarily labeled positive, and counterclockwise rotations are negative.

changes in vivo. This, in turn, renders impossible studies of the natural history and response to therapy of this disease.

In continuing this investigation, we observed a curvature dependence to the measured backscattered pressure field as a function of angle. Therefore, a potential problem exists in studying surface fibrillatory changes in osteoarthritic articular cartilage that depends on the ability to detect surface fibrillations on curved surfaces. To study this dependence, we used phantoms composed of acrylic rods with and without sandpaper grit applied to the surface. Experiments were performed using different radii of curvature and varying sandpaper grits. The results were compared to a flat, aluminized laser-grade, optical mirror. Two 6.4 $\mathrm{mm}$ diameter active element, $5 \mathrm{MHz}$ broad banded transducers, focused and unfocused, were employed in the experiments.

A more robust technique than that used in our previous work was used to measure the backscattered acoustic wave as a function of incident angle. The mean power (integrated squared pressure amplitude over a finite time window) was measured as a function of polar angle (angle of transducer measured relative to the surface normal, Fig. 1). The backscattered power provided excellent discrimination between varying surface roughness in phantoms, but the potential dynamic range (the relative change in backscattered power from normal incidence to the off-normal asymptotic plateau) for making these determinations diminished with decreasing radius of curvature of the acrylic rod phantoms when using an unfocused transducer. We demonstrate herein that finite curvature effects may be largely circumvented utilizing focused transducers, and that roughness effects are most evident at sufficiently large angles where incoherent scattering dominates.

\section{EXPERIMENTAL EQUIPMENT}

Figure 2 shows a block diagram description of the experimental equipment. Four primary components are used: a positioning system, an ultrasonic pulser/receiver unit with a General Purpose Interface Bus (GPIB), an IBM-compatible computer (PC) controlling the pulser/receiver unit and a SUN computer workstation with MATLAB for processing the acquired data. MATLAB (The Mathworks Inc., South Natick, MA) is a mathematical software package that performs numerical, matrix-oriented computations and data plotting.

The positioning system holds the sample in place and positions the ultrasound transducer for rotation around the sample (see Fig. 3). The primary components of the positioning system consist of optical bench components from Oriel Corp (Stratford, CT). The positioning system is divided into four components: a transducer polar angle rotator assembly, a sample tilt table, an alignment laser and an optical rail bench. The transducer polar angle rotator assembly provides $\mathrm{XYZ}$ positioning and polar angle rotation of the ultrasound transducer around the sample. The transducer is mounted in a gimbal mount on an armature attached to angular rotator of the assembly. The sample tilt table primarily allows the sample and its container to be aligned in the $\mathrm{X}, \mathrm{Y}$, or $\mathrm{Z}$ orientations independent of the transducer rotator assembly, allowing the sample surface to be positioned normal to the transducer face. A low power, $1 \mathrm{~mW}$ helium-neon laser (Oriel Corp.) is used to provide linear alignment of the sample to be scanned with the center of rotation of the polar angle rotator. The optical bench provides macro linear positioning of the other three components, which sit on top of sliders that mate with the bench. All these components together provide multiple degrees of freedom and precision alignment of the transducer and the sample.

The ultrasound pulser/receiver unit is a custom modified SRL 1712B CUI (Computerized Ultrasonic Instrument, System Research Laboratory, Dayton, $\mathrm{OH})$. It consists of a tunable pulser, a $50 \mathrm{MHz} 8$-bit digital receiver and an angular decoder attachment that 


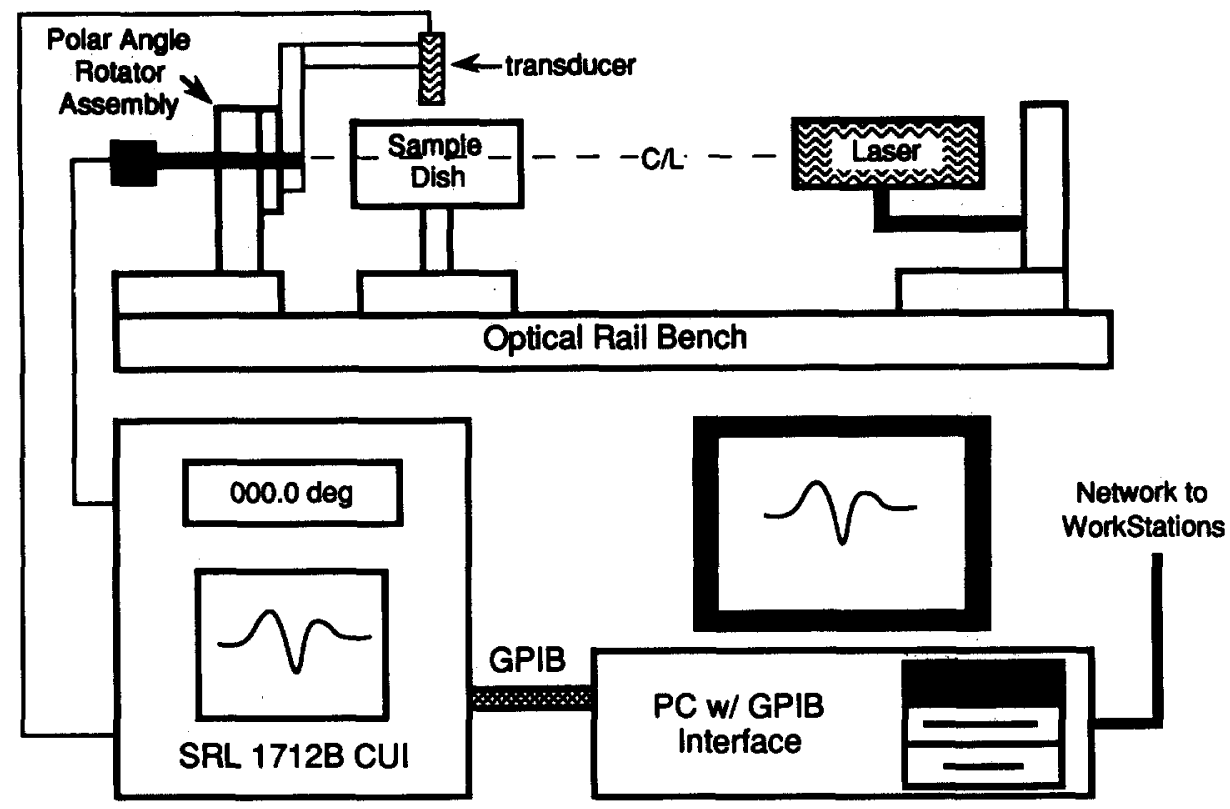

Fig. 2. Block diagram showing the major components of the Ultrasound Surface Roughness Data Acquisition System.

displays angular rotation with a resolution of 0.1 degrees. The unit is capable of capturing both raw, radiofrequency (RF) A-mode and B-mode data. This data may be stored on custom floppy disks or off loaded to a host computer using GPIB. Built-in functions of the SRL CUI include continuous signal averaging of Amode scans, and computation of A-mode spectrums. The unit is controlled either directly through the front panel controls, or by remote commands from a host computer sent through GPIB interface. An IBM-compatible PC with a GPIB interface running a customwritten program is used to control the SRL CUI during data acquisitions.

The two ultrasonic transducers used are both 5 $\mathrm{MHz}$ units with $6.4 \mathrm{~mm}$ diameter active elements (Panametrics Inc., Waltham, MA). The focused transducer has a measured center frequency of $5.05 \mathrm{MHz}$ with a $-6 \mathrm{~dB}$ bandwidth of $135 \%$, and is focused at a distance

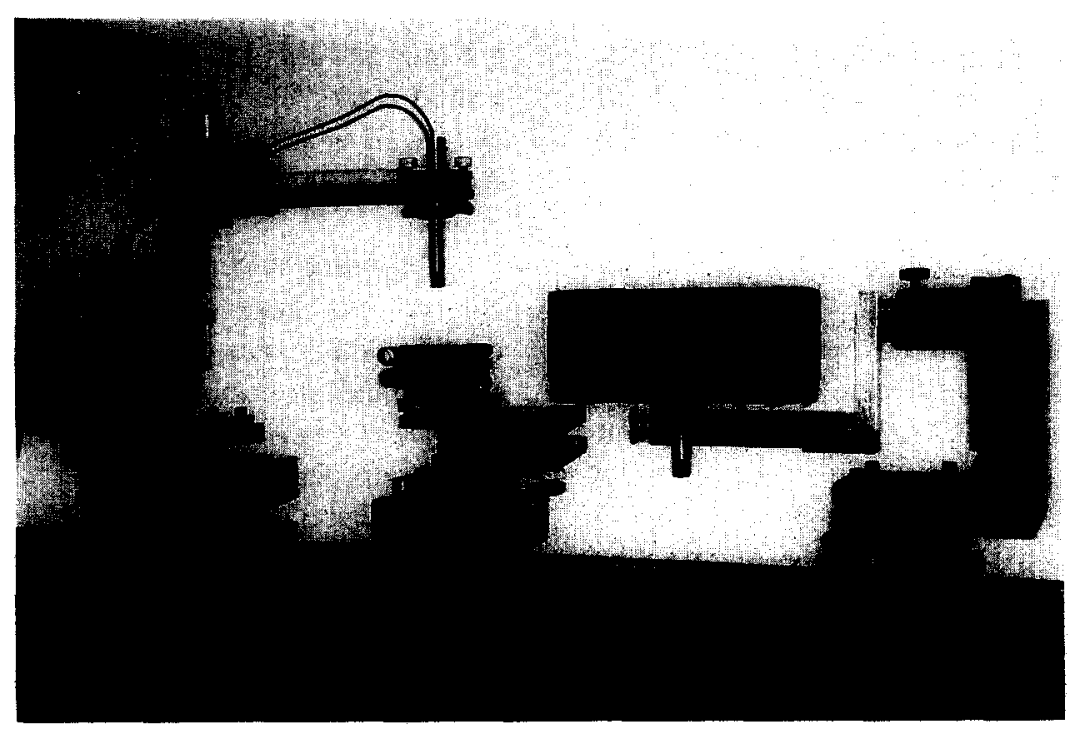

Fig. 3. Photograph of the positioning system used to align the ultrasonic transducer with the sample (shown without the sample dish in place). 


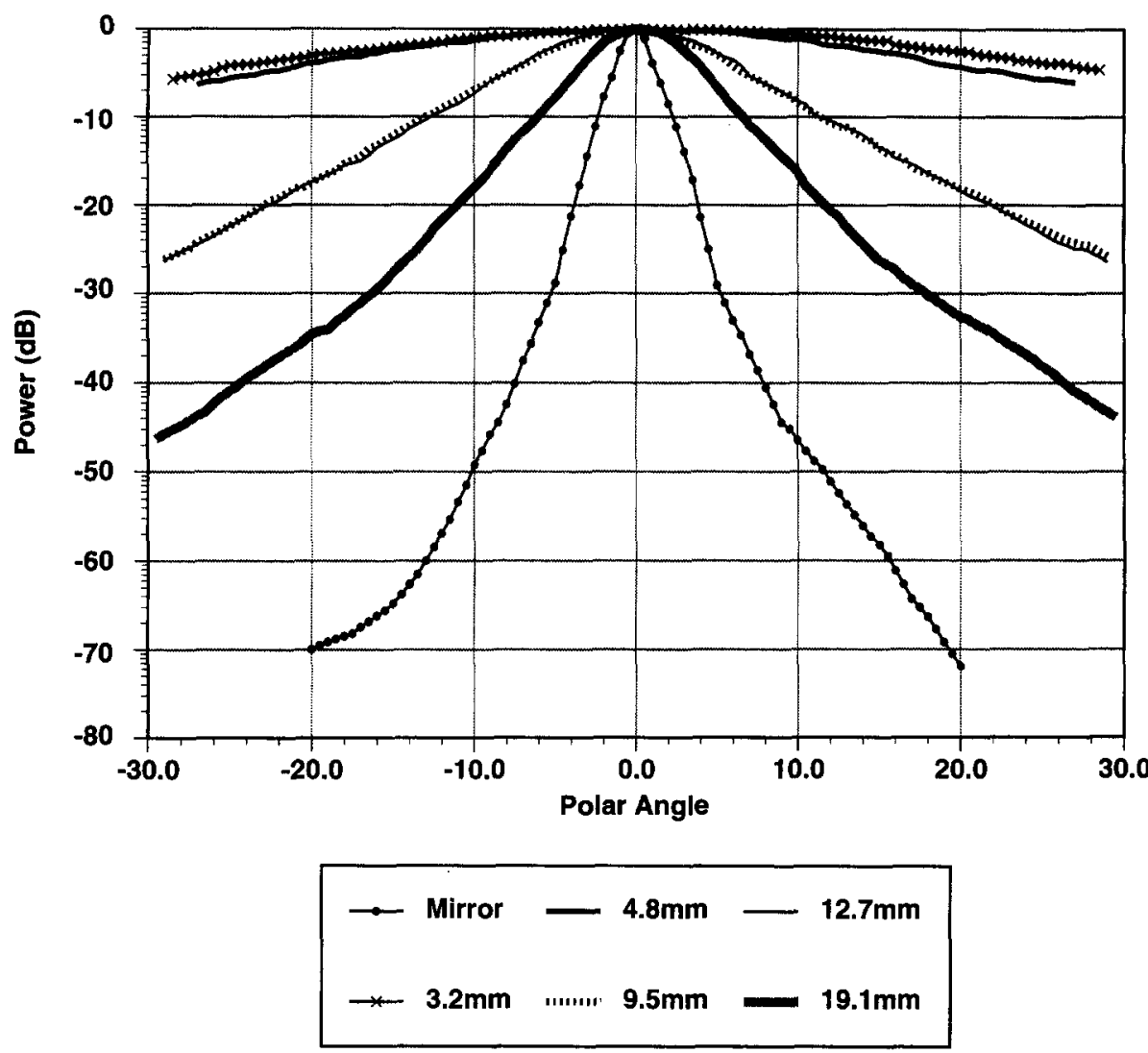

Fig. 4. Backscattered acoustic power from the smooth acrylic rod phantoms of varying radii of curvature using a $5 \mathrm{MHz}$ unfocused transducer. The backscattered power from a flat, optical mirror is also plotted as a reference. The backscattered power from the $9.5 \mathrm{~mm}$ and $12.7 \mathrm{~mm}$ radii phantoms overlap.

of $16 \mathrm{~mm}$ with a spot size diameter of $0.80 \mathrm{~mm}$ at the $-6 \mathrm{~dB}$ (Transmit-Receive amplitude) isocontour. The unfocused transducer has a measured center frequency of $5.7 \mathrm{MHz}$ with a $-6 \mathrm{~dB}$ bandwidth of about $123 \%$, and has a spot size diameter of $2.4 \mathrm{~mm}(-6 \mathrm{~dB}$ TR amplitude isocontour), past the far field transition point at a distance of $35 \mathrm{~mm}$.

\section{EXPERIMENTAL PROCEDURES}

Twenty-five millimeter long sections of solid acrylic rods in five arbitrarily selected radii were used: $3.2 \mathrm{~mm}, 4.8 \mathrm{~mm}, 9.5 \mathrm{~mm}, 12.7 \mathrm{~mm}$ and $19.1 \mathrm{~mm}$. The $9.5 \mathrm{~mm}, 12.7 \mathrm{~mm}$ and $19.1 \mathrm{~mm}$ radius rods were cut in half lengthwise. The $12.7 \mathrm{~mm}$ and $19.1 \mathrm{~mm}$ radius rods have radii that approximate the radius of curvature found in human femoral heads and femoral condyles.

For the "rough acrylic rod phantom" scans, the acrylic rods had a specific sandpaper grit glued to the acrylic surface. The sandpaper grit was glued to the cylindrical surface of the acrylic rods using spray mount glue. After drying, any excess sandpaper grit was removed. The acrylic rod phantoms, when scanned, were oriented with their longitudinal axes horizontal and perpendicular to the scan plane. For the $3.2 \mathrm{~mm}$ and the $4.8 \mathrm{~mm}$ radius acrylic rod phantoms, a short standoff was used to raise them off the bottom of the glass sample dish. The sample dish was then secured to the sample tilt table of the positioning system.

Degassed water was used during all acquisitions. One or two drops of Kodak Photo-Flo 200 solution were added to the degassed water to reduce surface tension and air bubble formation. If the phantom was "rough," it was occasionally brushed when underwater to remove any air bubbles clinging to the rough surface. Bubbles clinging to the rough surface could erroneously indicate a smoother surface. The degassed water was added during the sample alignment process (see below).

Before each set of scans, the positioning system was put through an alignment procedure to orient the ultrasound transducer normal to the sample tilt table. The alignment was performed by using a carbon tetra- 

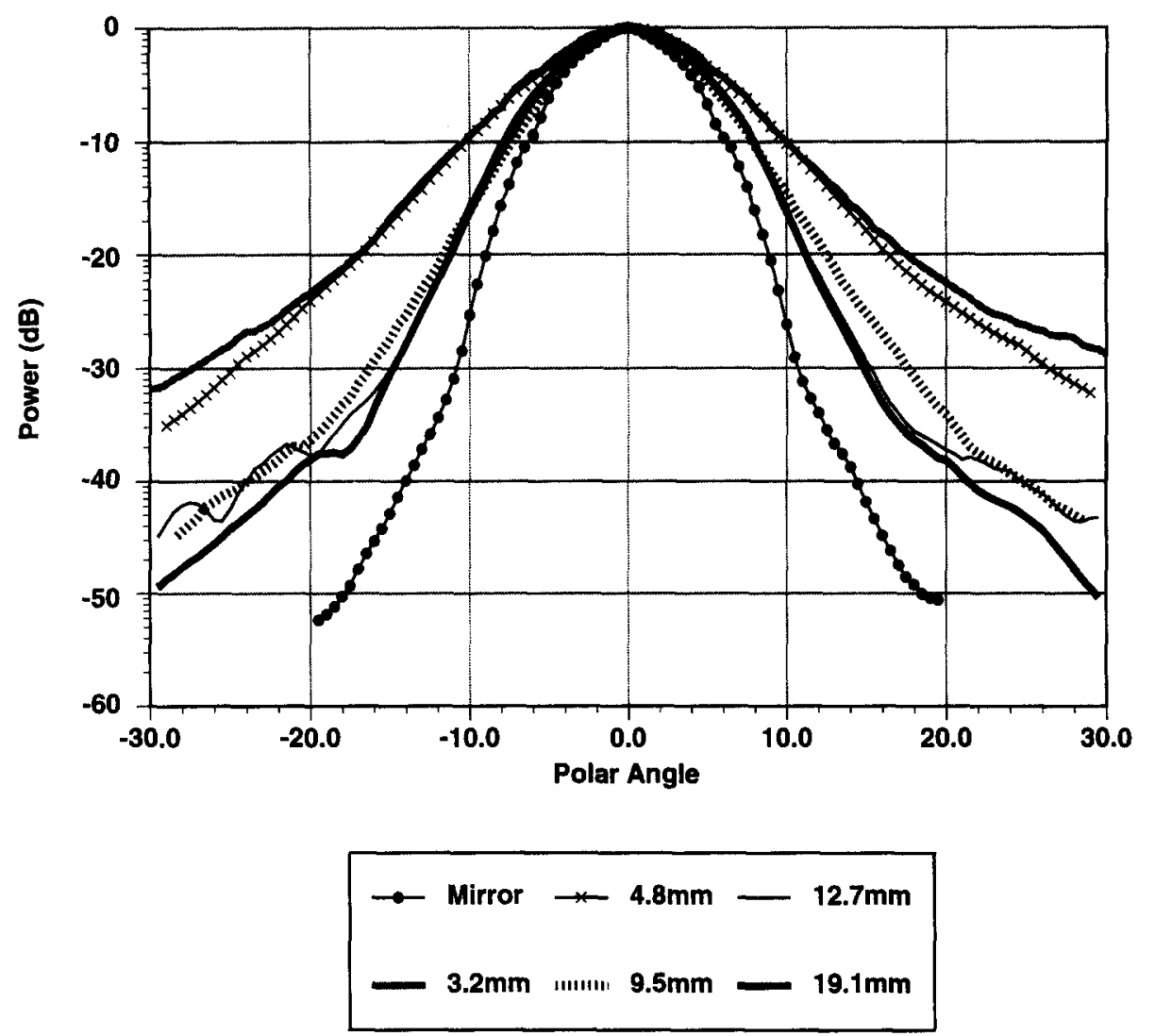

Fig. 5. Backscattered acoustic power from the smooth acrylic rod phantoms of varying radii of curvature. In this case a $5 \mathrm{MHz}$ focused transducer was used.

chloride $\left(\mathrm{CCl}_{4}\right) /$ water interface. Because $\mathrm{CCl}_{4}$ is immiscible in and denser than water, the interface between the two liquids provided an ideally flat surface. The gimbal mount attached to the transducer adjusted the transducer orientation (tilt) and allowed normal orientation to the sample tilt table to be maintained. This alignment helped compensate for any mechanical misalignments within the positioning system.

Surface alignment of the sample was then performed relative to the center of rotation of the polar angle rotator prior to each acquisition. The alignment laser was used to ensure that the sample surface was tangent to the axis of rotation. This was undertaken as a two-step procedure prior to the addition of degassed water and Photo-Flo. First, before degassed water was added to the sample dish and with the dish on top of the sample tilt table, the alignment laser was translated vertically so that its horizontal light beam touched the surface of the sample to be scanned. Next, the sample dish (with the sample inside) was removed from the sample tilt table. The transducer polar angle rotator assembly was translated vertically so that the center of rotation of the polar angle rotator was in line with the beam of the alignment laser. At this point the sample dish was replaced and the degassed water and Photo-Flo added. This two-step alignment procedure was performed to avoid the refraction of the laser light through the water in the sample dish. A slight refraction of laser light remained because of the glass sample dish.

The final step before data acquisition was positioning the transducer vertically (at $0.0^{\circ}$ polar) above the sample with its focal point at the sample surface. This was done using the vertical slider and the attached micro translator of the transducer armature, because the vertical position of the center of the polar angle cannot be changed. The sample was aligned to maximize the backscattered echo at the transducer. The alignment was accomplished using the translators on the tilt table and the tilt table itself. Any minor adjustments of the polar angle rotator were also made at that time, usually within $\pm 2^{\circ}$. This additional alignment compensated for any mechanical misalignments of the positioning system.

RF A-mode lines were acquired by the SRL CUI at $0.5^{\circ}$ polar angle intervals. The polar angle rotator 


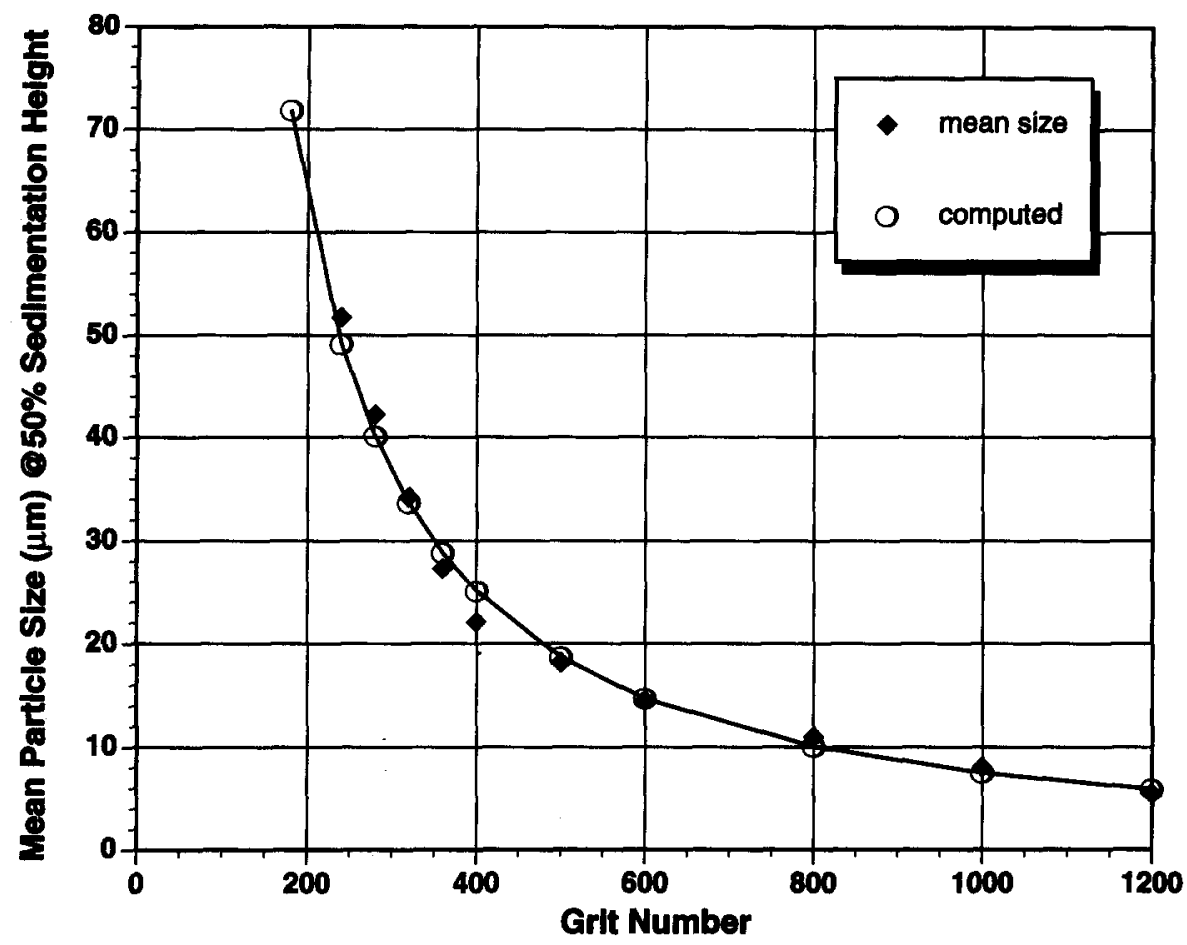

Fig. 6. Graph of mean particle size with corresponding grits as measured by sedimentation in ANSI Standard B74.10 using the $50 \%$ column height. Grit number and mean particle size were used to determine the empirical formula and the computed results.

was rotated by hand using the digital readout from the SRL CUI as a guide. Each A-mode line acquisition was downloaded and stored on the PC using the GPIB interface between the PC and SRL CUI. The associated receiver gain and polar angle were recorded in a text file on the PC.

A-mode line acquisitions started at $0.0^{\circ}$ polar and continued in one angular direction either positive $\left(+0.5^{\circ}, 40.0^{\circ}\right)$ or negative $\left(-0.5^{\circ},-40.0^{\circ}\left[395.5^{\circ}\right.\right.$, $\left.320.0^{\circ}\right]$ ). Clockwise rotations were arbitrarily considered positive polar angles (see Fig. 1). Once one angular direction was completed, the rotator was swept back to $0.0^{\circ}$ and data in the other angular direction was acquired. There was minimal backlash in the angular rotator. The actual angular sweep range was limited by the height of the sides of the glass sample dish and the depth with which the transducer extended into the sample dish.

The SRL CUI pulser/receiver provided different length acquisition windows or ranges. Generally, the smallest range of $1.5 \mu \mathrm{s}$ was used. The receiver delay time of the SRL CUI was adjusted to place the initial backscattered echo at the center of the acquisition window. At increased incident angles, care was taken to ensure that only the echo from the phantom surface was within the acquisition window. Misalignment of the polar angle rotator with the sample surface appeared as movement of the backscattered echo within the acquisition window, and a change in echo delay time.

\section{ANALYSIS}

The preliminary step in the data analysis was reformatting the individual A-mode data into a single MATLAB readable (".mat") file. The actual analysis began with the computation of the normalized power at each polar angle,

$$
P(\theta)=\frac{1}{P_{0}} \int_{T}\left[Q_{g}(\theta), V(\theta, t)\right]^{2} d t,
$$

where

$v(\theta, t)$ is the amplitude of the A-mode signal at time $t$ for polar angle $\theta$;

$T$ is the acquisition window length;

$Q_{g}(\theta)$ is the receiver gain normalization factor for polar angle $\theta$ - see eqn (2); and

$P_{0}$ is the peak power-see eqn (3). 


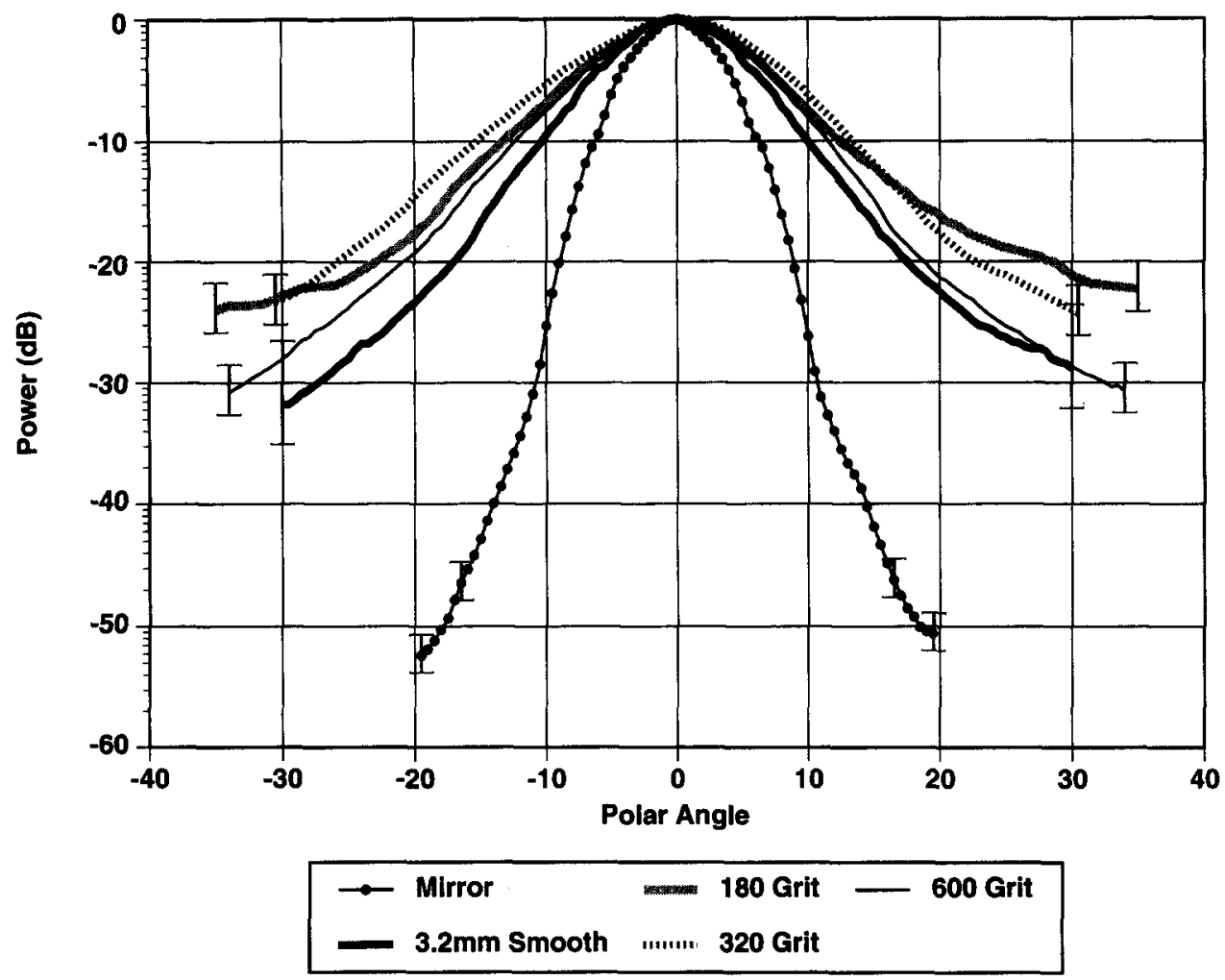

Fig. 7. Figures 7 to 11 show the backscattered acoustic power from differing radii acrylic rod phantoms with varying sandpaper grits. All the phantoms were scanned using a $5 \mathrm{MHz}$ focused transducer. The backscattered power from a smooth optical mirror and the corresponding smooth acrylic rod are also plotted. Ninety-five percent confidence limits are included for each power.

The differing receiver gains used for each polar angle were accounted for by using the receiver gain normalization factor,

$$
Q_{g}(\theta)=\log _{10}^{-1}\left(-\frac{G(\theta)-G_{\min }}{20}\right)
$$

where

$G_{\text {min }}$ is the predetermined minimum receiver gain in decibels; and

$G(\theta)$ is the receiver gain at polar angle $\theta$ in decibels.

All backscattered powers were normalized to the peak power,

$$
P_{0}=\int_{T}\left[Q_{g}(\varphi) V(\varphi, t)\right]^{2} d t
$$

where $\varphi$ is the polar angle at peak power, usually $0.0^{\circ}$.

Using MATLAB, the backscattered powers were computed within an acquisition data set. The peak power was expected to occur at zero degrees polar; however, a displacement of $\pm 1.0^{\circ}$ was not uncommon.
If the peak power did not occur at zero degrees, then a translation of the data was performed to shift the acquisition data set so that peak power was centered at zero degrees polar. The data set was truncated so that it was symmetric about this zero degree, peak power point.

The integration was done numerically using Simpson's rule, because it provided better integration results than trapezoidal based integration, yet, was still simple to implement. An acquisition window of $1.5 \mu \mathrm{s}$ was used, which is the smallest window available on the SRL CUI. The backscattered echo from the insonified sample was windowed within the $1.5 \mu$ s so that only the signal from the desired surface (generally, the sandpaper grit) was acquired. Acquisition of echoes from any underlying structures was kept to a minimum. Furthermore, experiments using a larger acquisition window of $3.0 \mu$ s showed no significant power difference compared to the $1.5 \mu \mathrm{s}$ window. At the $50 \mathrm{MHz}$ digitization rate of the SRL CUI pulser/receiver, the $1.5 \mu \mathrm{s}$ acquisition window corresponded to 75 data points per A-mode line. Individual A-mode lines were acquired over a range of approximately $\pm 40^{\circ}$. The exact polar angle range was dependent upon experimental condi- 

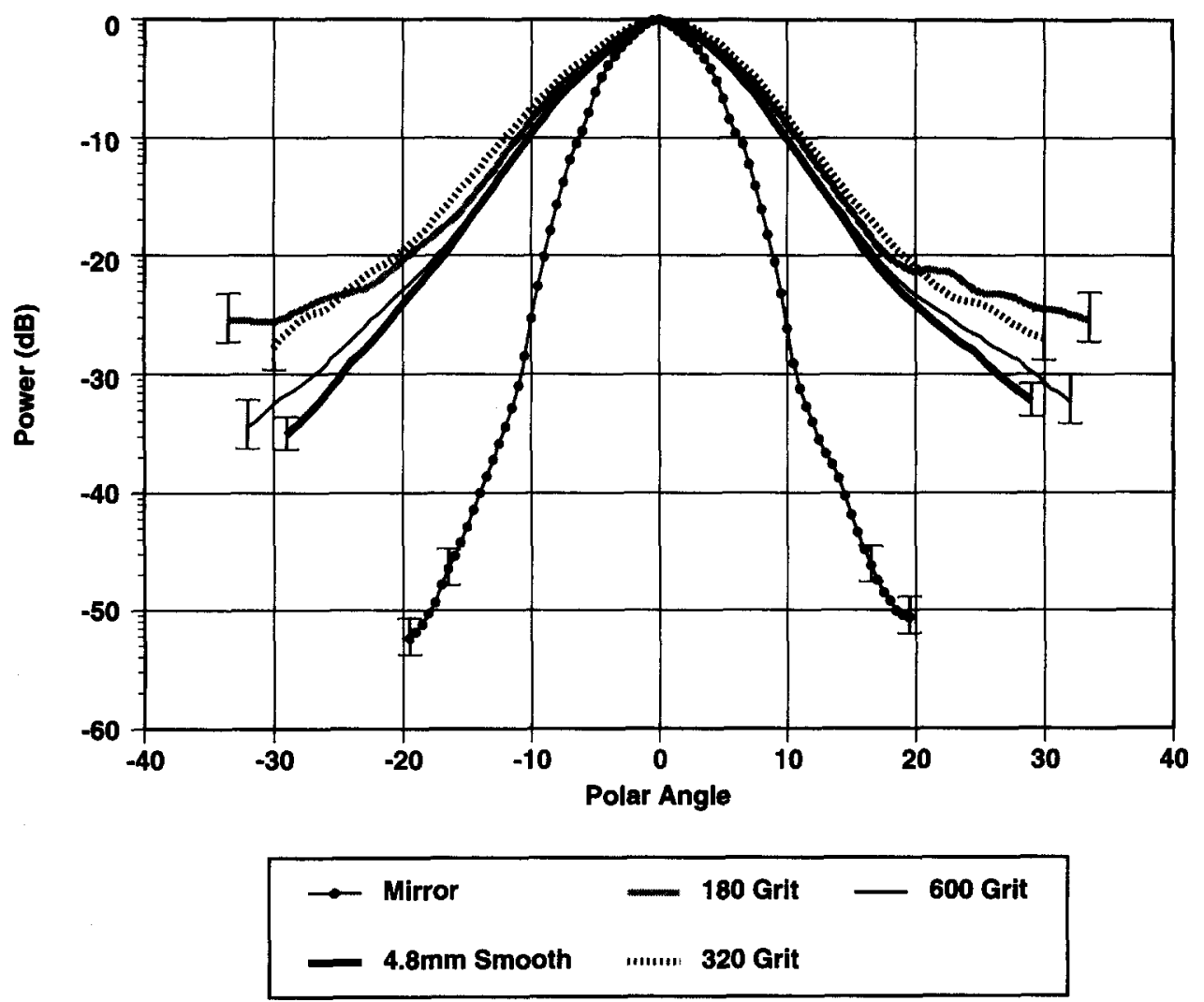

Fig. 8. See legend to Fig. 7.
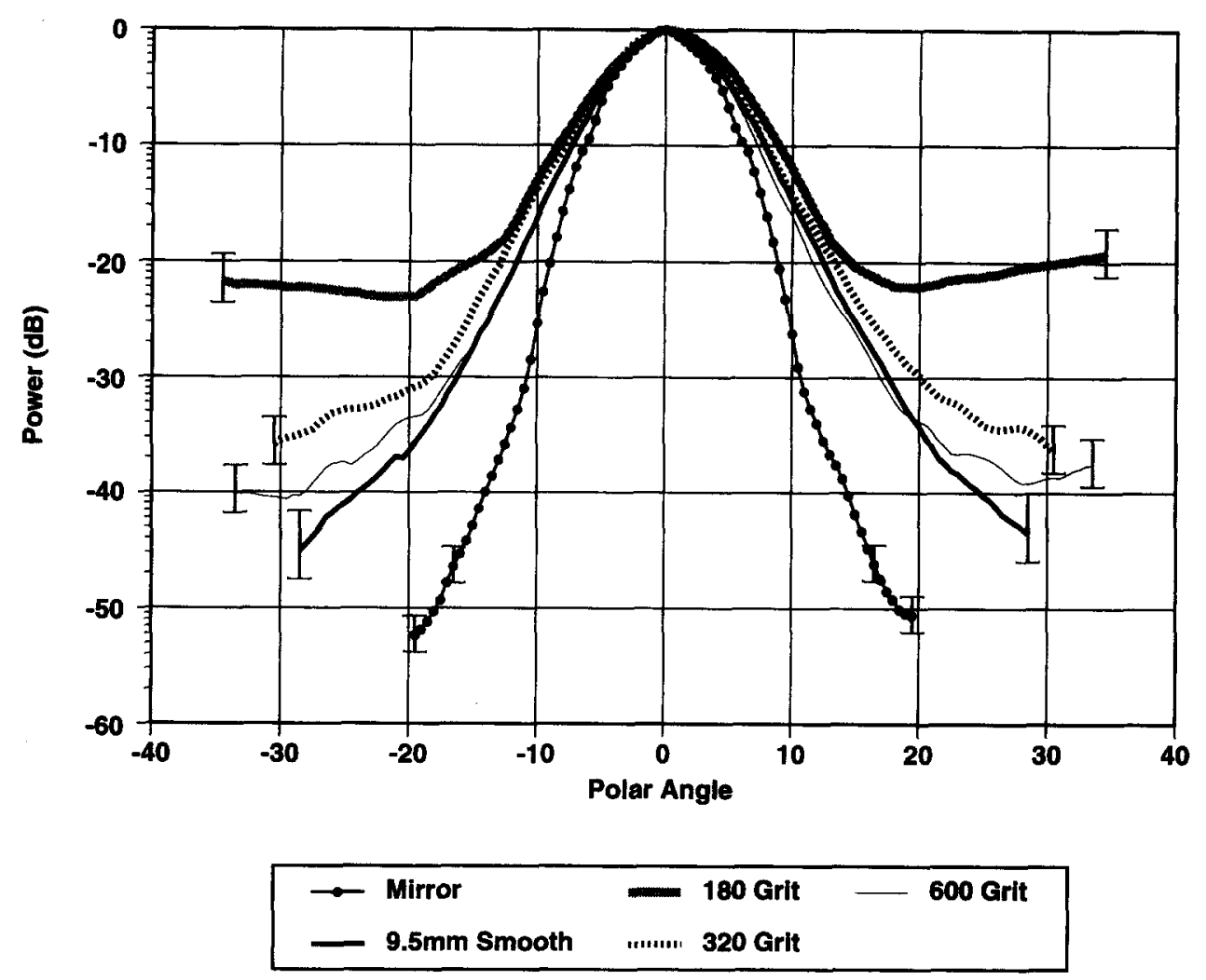

Fig. 9. See legend to Fig. 7. 

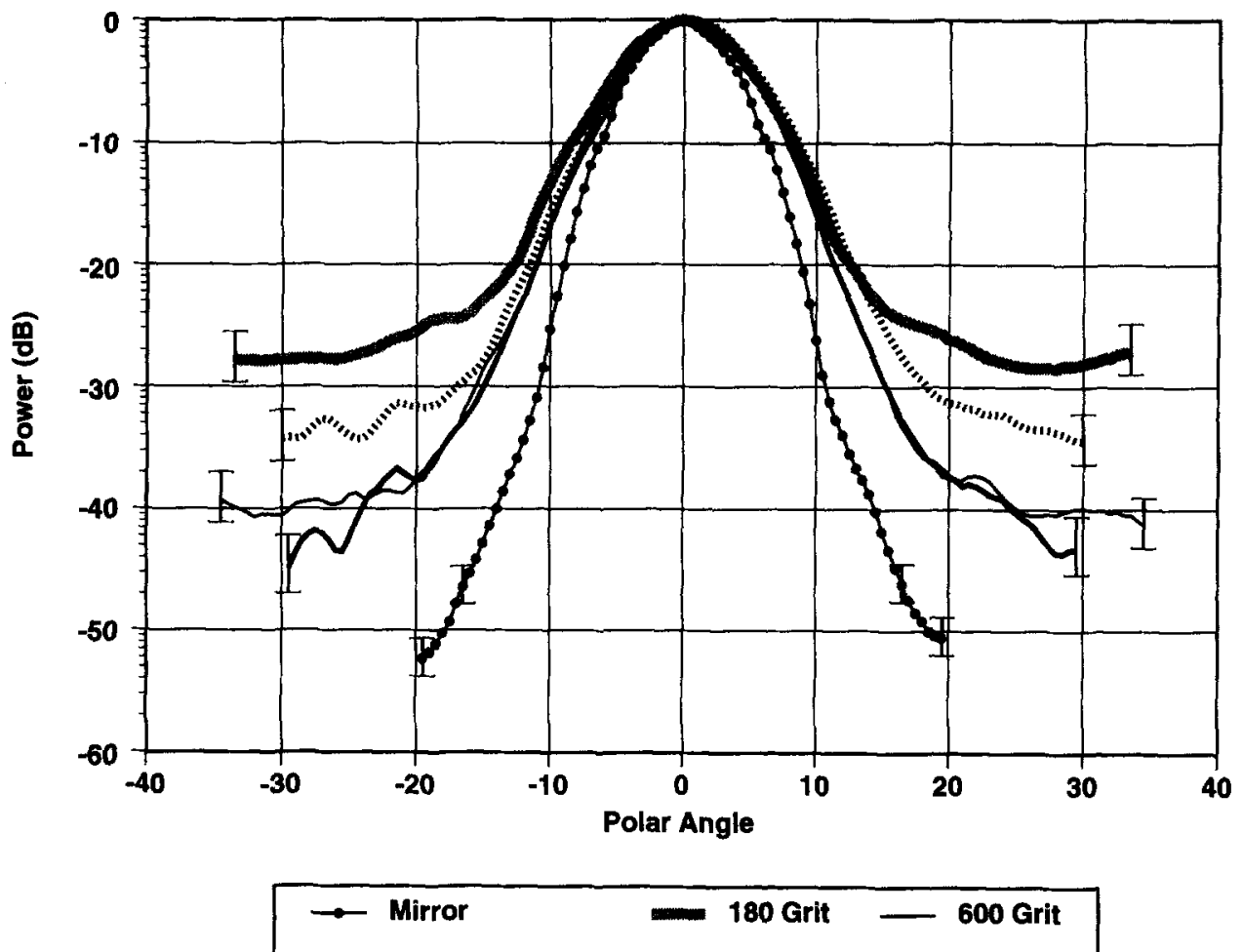

- 12.7mm Smooth 1un! 320 Grit

Fig. 10. See legend to Fig. 7.

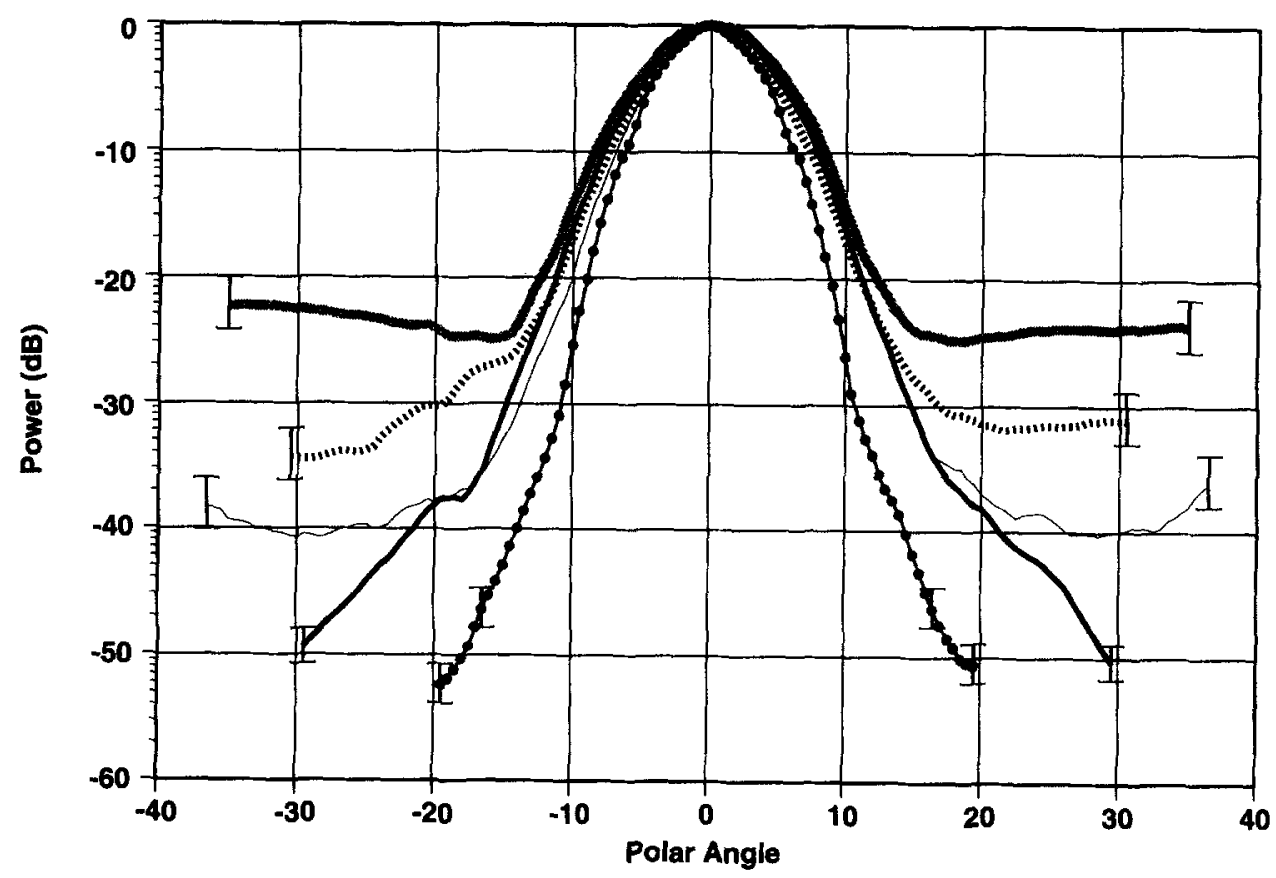

\begin{tabular}{|lll}
\hline$\rightarrow-$ Mirror & -180 Grit -600 Grit \\
$-19.1 \mathrm{~mm}$ Smooth & $\cdots \cdots+320$ Grit
\end{tabular}

Fig. 11. See legend to Fig. 7. 
tions. The resultant data were plotted in a semilog fashion. The normalized backscattered power was plotted logarithmically on the ordinate with a peak value of 1 , and the corresponding polar angle was plotted on the abscissa. The results were generally symmetric about $0.0^{\circ}$ polar, appearing as a "bell" shaped curve.

\section{RESULTS}

Results for both the focused and unfocused transducers insonifying the acrylic rod phantoms are shown in Figs. 4 and 5. For comparison, a flat, $25.4 \mathrm{~mm}$ diameter, aluminized laser-grade optical mirror was also insonified by both the unfocused and focused transducers. The backscattered power as a function of angle was measured from the mirror. The minimum receiver gain used with the mirror was then used as the receiver gain normalization factor, $G_{\min }$ in eqn (3). In the case of the unfocused transducer $G_{\min }$ was -4 $\mathrm{dB}$, and in the case of the focused transducer, $-7 \mathrm{~dB}$.

As expected, the dynamic range (the relative change in backscattered power from normal incidence measured with respect to the asymptotic plateau) of the backscattered power from the smooth (no grit) acrylic rod phantom, when using the unfocused $5 \mathrm{MHz}$ transducer, was highly dependent upon radius of curvature. (Fig. 4.) The spot size diameter ( $-6 \mathrm{~dB}$ TR amplitude isocontour) of the unfocused transducer was 2.4 $\mathrm{mm}$ at a distance of $35 \mathrm{~mm}$, which is past the far field transition point. For each of the five phantoms, two scans were performed and the resultant backscattered powers averaged. For objects with a radius of curvature similar to the transducer spot size, as in the $3.2 \mathrm{~mm}$ and $4.8 \mathrm{~mm}$ radius phantoms, there was very little change in backscattered power as a function of polar angle. The dynamic range in these cases was less than $10 \mathrm{~dB}$ at $\pm 28^{\circ}$. One would expect that backscattered power as a function of angle from a point source would be constant for all angles, having a dynamic range of 0 $\mathrm{dB}$. With increasing radius of curvature, corresponding increases in dynamic range of the backscattered power became evident. For example, the $9.5 \mathrm{~mm}$ and 12.7 $\mathrm{mm}$ radius phantoms had a dynamic range of about 29 $\mathrm{dB}$ at $\pm 28^{\circ}$. The dynamic range increased further to $49 \mathrm{~dB}$ for the $19.1 \mathrm{~mm}$ radius phantom. The useful dynamic range to determine surface roughness, using an unfocused transducer, diminished with decreasing radius of curvature. Because of this curvature dependence, roughness experiments were performed with focused transducers to reduce the insonified spot size.

Using the focused transducers, the backscattered power as a function of angle from the smooth acrylic rod phantoms was less sensitive to radius of curvature
Table 1. Particle size with corresponding grits as measured by sedimentation in ANSI Standard B74.10 using the $50 \%$ column height.

\begin{tabular}{rrrrrr}
\hline & \multicolumn{5}{c}{$50 \%$ Height sedimentation } \\
\cline { 2 - 6 } & \multicolumn{5}{c}{ Particle size in microns } \\
\cline { 2 - 6 } Grit & Max & Min & Mean & Computed & \% Error \\
\hline 180 & - & - & - & 71.8 & - \\
240 & 53.5 & 50.0 & 51.75 & 49.1 & 5.1 \\
280 & 44.0 & 40.5 & 42.25 & 40.1 & 5.1 \\
320 & 36.0 & 32.5 & 34.25 & 33.6 & 1.8 \\
360 & 28.8 & 25.8 & 27.30 & 28.8 & 5.5 \\
400 & 23.6 & 20.6 & 22.10 & 25.1 & 13.4 \\
500 & 19.7 & 16.7 & 18.20 & 18.7 & 2.6 \\
600 & 16.0 & 13.0 & 14.50 & 14.7 & 1.3 \\
800 & 12.3 & 9.8 & 11.05 & 10.1 & 9.0 \\
1000 & 9.3 & 6.8 & 8.05 & 7.5 & 6.9 \\
1200 & 6.5 & 4.5 & 5.50 & 5.9 & 7.2 \\
\hline
\end{tabular}

Grit number and mean particle size were used to determine the empirical formula (eqn 4). Computed results and errors for this formula are also shown in the table. Grit sizes 180,320 and 600 grit were used in acrylic rod phantoms. The mean particle size for 180 grit was computed using the empirical formula.

effects (Fig. 5). The focused $5 \mathrm{MHz}$ transducer, in this case, had a spot size diameter $(-6 \mathrm{~dB}$ TR amplitude isocontour) of $0.80 \mathrm{~mm}$ at the focus, which occurs at $16 \mathrm{~mm}$. For each of the five acrylic rod phantoms, four separate scans were performed and the resultant backscattered powers averaged. The resulting plot showed a similar dependence on radius of curvatures with the unfocused transducer case. However, the dynamic range was significantly larger. The dynamic range was about $42 \mathrm{~dB}$ at $\pm 30^{\circ}$, which differed from the optical mirror at $\pm 20^{\circ}$ by approximately $25 \mathrm{~dB}$ in backscattered power. This difference was less pronounced in the case of the larger radius acrylic rod phantoms, which showed only about a $15 \mathrm{~dB}$ difference in backscattered power relative to the optical mirror. In general, the dynamic range for backscattered power as a function of polar angle was much larger and more consistent using a focused rather than unfocused transducer. The dynamic range of the focused transducer, independent of radius of curvature, was about $48 \mathrm{~dB}$ at about $\pm 30^{\circ}$.

Next, the backscattered power as a function of polar angle from grit covered acrylic rod phantoms was measured. Three grit sizes were used: 180 grit, 320 grit and 600 grit. The corresponding particle sizes as defined using the American National Standards Institute (ANSI) standard ANSI B74.10 are 72, 34 and $15 \mu \mathrm{m}$, respectively (see Table 1). Grit size 180 was not defined in the ANSI standard. Therefore, the particle size was extrapolated from the ANSI standard data using the empirical formula, 

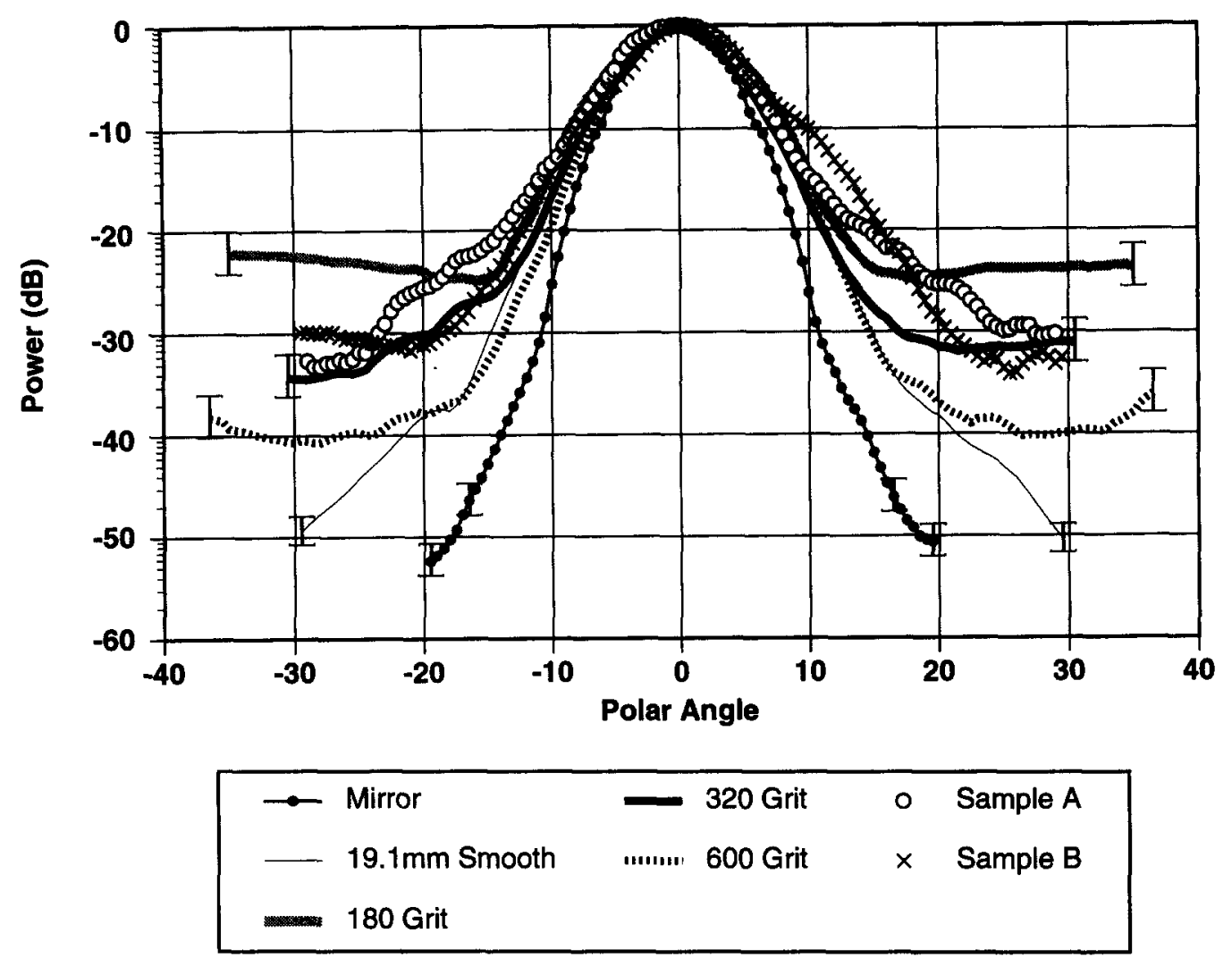

Fig. 12. Preliminary results of the backscattered power from two samples of grossly normal cartilage from a human femoral condyle. They are shown compared with the $191 \mathrm{~mm}$ radius phantoms.

$$
S(g) \approx\left(6.72 \times 10^{4}\right) g^{-1.32}\left(r^{2}=0.991\right)
$$

where

$g$ is the grit number; and

$S$ is the particle size in micrometers.

Equation 4 was derived using the curve fitting features of DeltaGraph ${ }^{\circledR}$ Professional (DeltaPoint, Monterey, CA) for the Macintosh computer. It was selected based on best $r^{2}$ (coefficient of determination) fit (see fig. 6).

Each sandpaper grit size was glued to the five acrylic rod phantoms, and the resulting rough phantoms insonified by the $5 \mathrm{MHz}$ focused transducer. The angle-dependent backscattered power, shown in Figs. $7-11$, illustrates that the power, as a function of angle and for a given radius of curvature, was greater for rougher surfaces or larger grit sizes. Conversely, the backscattered power at larger angles was expected to be less for smoother surfaces. Smooth surfaces were predicted to have backscattered power profiles similar to that of the mirror. Each backscattered power profile consisted of the ensemble mean of four acquisitions. Ninety-five percent confidence limits for the power are indicated for each plot. For the two smaller radii of curvature, $3.2 \mathrm{~mm}$ and $4.8 \mathrm{~mm}$, no really distinguishable differences in grit size (roughness) were seen in the backscattered power as a function of angle. In fact, the 320 grit appears to have more backscattered power for a given angle, implying that $\mathbf{3 2 0}$ grit is rougher. A more consistent backscattered power profile can be seen with the larger radii of curvature phantoms: 9.5 $\mathrm{mm}, 12.7 \mathrm{~mm}$ and $19.1 \mathrm{~mm}$. These plots illustrate the expected monotonic behavior of the backscattered power at a given polar angle with respect to surface curvature and smoothness. At sufficiently large angles, a plateauing effect was evident, the magnitude of which differs for each grit size and corresponding surface roughness. This effect is most apparent at the largest radius of curvature, $19.1 \mathrm{~mm}$.

\section{DISCUSSION}

The backscattered intensity of electromagnetic waves from a rough surface is typically separated into coherent and incoherent contributions, where intensity, $I$, is defined as power per unit area, or 

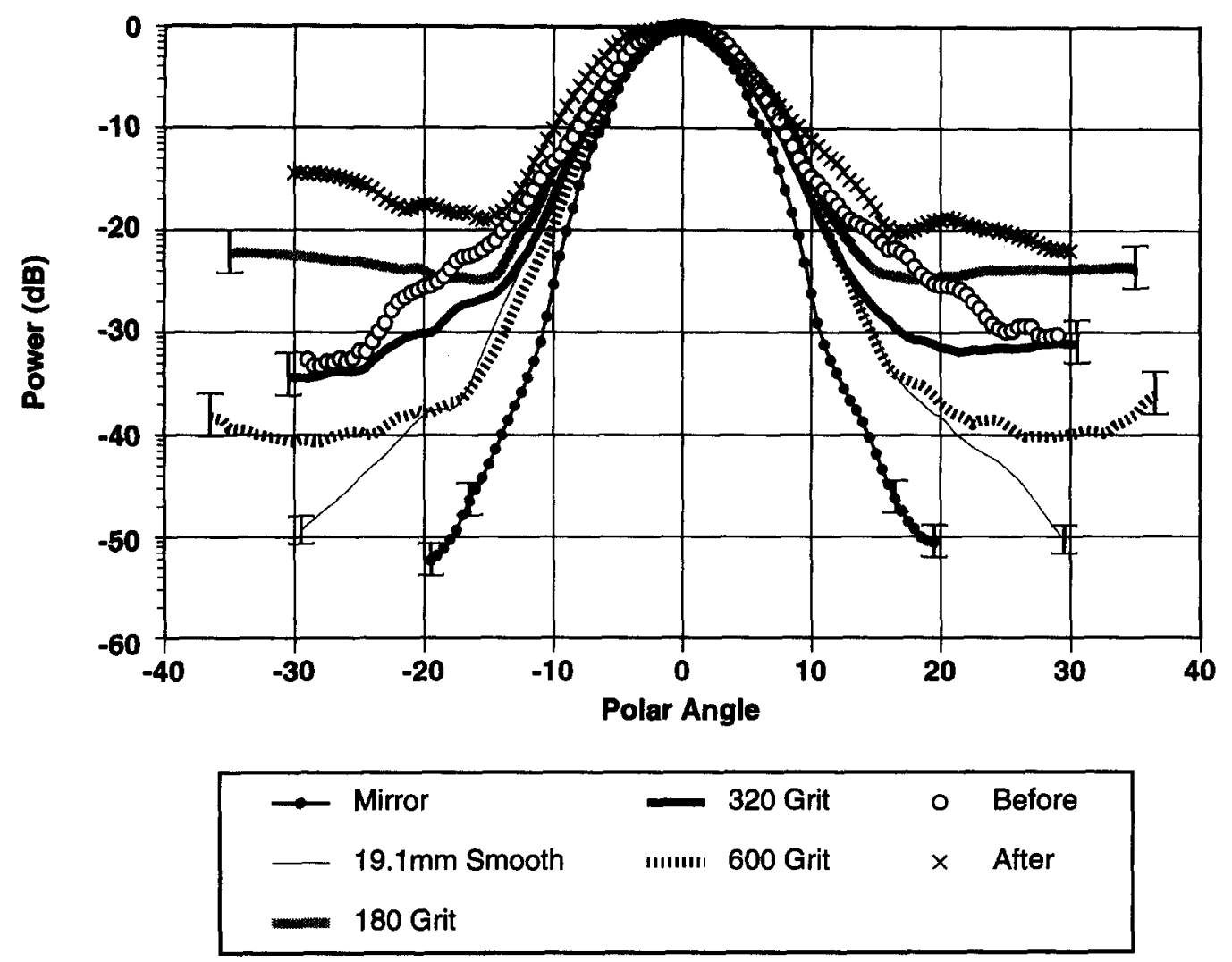

Fig. 13. The backscattered power after one of the femoral condyle samples was abraded with 100 grit emory paper.

$$
\langle I\rangle=\langle I\rangle_{\text {coherent }}+\langle I\rangle_{\text {incoherent }} .
$$

This also applies to acoustic waves. For slightly or moderately rough surfaces, the scattering is largely coherent and primarily specular. For rougher surfaces, incoherent scattering dominates in all directions (Bozma and Kuc 1991). Because the mirror is a smooth surface, the backscattered power (intensity) is largely coherent. This is also true of the smooth (no grit) acrylic rod phantoms. For the rough (grit-covered) acrylic rod phantoms, the coherent component is still evident at incident angles near normal between about \pm 15 to $\pm 20^{\circ}$. However, at larger polar angles, the incoherent component dominates, and it is in this region where surface roughness is more easily differentiated.

In general, our results correlated well with the experimental results of other investigators. Bozma and Kuc (1991), using a $60 \mathrm{kHz}$ Polaroid transducer, showed that the backscattered power as a function of incident angle could discriminate between a rough styrofoam planar surface and a smoothed (flattened) aluminum foil sheet. Their experimental data showed only about a $2 \mathrm{~dB}$ difference from their theoretical predictions. Welton et al. (1972) showed similar experimental results. They insonified four rough planar surfaces at three different frequencies: 100,200 and $500 \mathrm{kHz}$. Three of the surfaces had differing root-mean-square (rms) heights, while the fourth had a different surface correlation length compared to the other three. Their data, particularly at $500 \mathrm{kHz}$, showed differing dynamic ranges corresponding to the differing surface roughnesses.

Because the ultimate goal of this work is to characterize articular cartilage roughness, we have included some preliminary, in vitro results (Figs. 12 and 13) from grossly normal cartilage specimens obtained from lateral and medial femoral condyles excised from a human cadaver. The two samples were preserved in buffered formalin, rehydrated and scanned while immersed in normal saline. Figure 12 shows the backscattered power profiles from the surface echo for "normal" cartilage, which shows surface scattering similar to 320 grit. In an experiment to determine the differential effect of roughening the smooth cartilage surface, one of the femoral condyles was abraded with a piece 
of 100 grit emory paper. The 100 grit was chosen to ensure that the cartilage surface was significantly roughened. Visual inspection of the cartilage surface verified this. The backscattered power from the roughened cartilage surface is shown in Fig. 13. In comparison to the preabrasion power profile, there is a change of $10 \mathrm{~dB}$ on one side and $20 \mathrm{~dB}$ on the other side. The difference is likely due to anisotropy in the roughening process. These preliminary results suggest that surface fibrillatory changes produce differences in angular backscatter according to the level of surface roughening. Additional experiments with histological correlations need to be performed.

\section{CONCLUSION}

Based on the results presented herein for acoustic scattering from rough acrylic rod phantoms, two conclusions can be drawn. First, a focused transducer with a sufficiently small focal spot is necessary to effectively eliminate finite radius of curvature effects in the study of the surface roughness on curved surfaces. Second, at sufficiently large incident angles relative to surface normal, the resultant backscattered power is largely due to incoherent scattering, resulting in a nearplateau dependence. Over the first 15 to 20 degrees polar in our experiments, the specular (coherent) component dominates. Scattering in this region is relatively independent of the degree of roughness, apart from mild broadening effects. Surface roughness is, therefore, best differentiated in our experiments at larger polar angles, greater than about $15^{\circ}$.

Based on the results presented herein, we have been able to discriminate differences in rms surface roughness on the order of $30 \mu \mathrm{m}$ in model experiments. This size range is well within the range of irregularities seen in histologic studies of articular cartilage affected by early osteoarthritis. Surface fibrillations occurring in the range of 20 to $150 \mu \mathrm{m}$ have been demonstrated in patients with osteoarthritis (Minns et al. 1977; Bland and Cooper 1984). Preliminary in vitro results sug- gested that the present technique may be applied to the study of surface fibrillatory changes present in osteoarthritic articular cartilage in vitro. Following in vitro validation for a large variety of $\mathrm{OA}$ affected articular cartilage, the methodology may be extended to in vivo applications, vis-à-vis a catheter based intraarticular system.

Acknowledgements - Portions of this research were supported in part by NIH Grant Numbers K11-ARO1793 and 5P60-20557. The authors would also like to thank Ronald Bude, M.D., for his help in acquiring, and the $3 \mathrm{M}$ Company for supplying, the sandpaper grit.

\section{REFERENCES}

Adler, R. S.; Dedrick, D. K.; Laing, T. J.; Chiang, E. H.; Meyer, C. R.; Bland, P. H.; Rubin, J. M. Quantitative assessment of cartilage surface roughness in osteoarthritis using high frequency ultrasound. Ultrasound Med. Biol. 18:51-58; 1992.

Bland, J. H.; Cooper, S. M. Osteoarthritis: A review of the cell biology involved and evidence for reversibility. Management rationally related to known genesis and pathophysiology. Sem. Arthritis Rheum. 14:106-133; 1984.

Bozma, O.; Kuc, R. Characterizing pulses reflected from rough surfaces using ultrasound. J. Acoust. Soc. Am. 89:2519-2531; 1991.

De Billy, M.; Cohen-Tenoudji, F.; Jungman, A.; Quentin, G. The possibility of assigning a signature to rough surfaces using ultrasonic backscattering diagrams. IEEE Trans. Ultrason. SU 23:356-363; 1976.

Lynch, P. J. Curvature corrections to rough-surface scattering at high frequencies. J. Acoust. Soc. Am. 47:804-815; 1970.

Minns, R. J.; Stevens, F. S.; Hardinge, K. Osteoarthritic articular cartilage lesions of the femoral head observed in the scanning electron microscopy. J. Pathol. 122:63-70; 1977.

Quentin, G.; De Billy, M.; Cohen-Tenoudji, F.; Doucet, J.; Jungman, A. Experimental results of the scattering of ultrasound by randomly and periodically rough surfaces in the frequency range 2 to $25 \mathrm{MHz}$. Ultrasonics Symposium Proceedings, IEEE Press, $102-106 ; 1975$.

Rayleigh, L. (Strutt J. W.) Theory of sound. New York: Dover; 1945.

Thorne, P. D.; Pace, N. G. Acoustic studies of broadband scattering from a model rough surface. J. Acoust. Soc. Am. 75:133-144; 1984.

Thorsos, E. I. The validity of the Kirchhoff Approximation for rough surface scattering using a gaussian roughness spectrum. J. Acoust. Soc. Am. 83:78-92; 1988.

Welton, P. J.; Frey, H. G.; Moore, P. Experimental measurements of the scattering of acoustic waves by rough surfaces. J. Acoust. Soc. Am. 52:1553-1563; 1972. 